\title{
"Deborah numbers", coupling multiple space and time scales and governing damage evolution to failure
}

\author{
Y.L. Bai ${ }^{\text {a }}$, H.Y. Wang ${ }^{\text {* }}$, M.F. Xia ${ }^{\text {b,a }}$, F.J. Ke $e^{\text {c,a }}$ \\ ${ }^{a}$ LNM, Institute of Mechanics, Chinese Academy of Sciences, Beijing 100080, China \\ ${ }^{\mathrm{b}}$ Department of Physics, Peking University, Beijing 100871, China

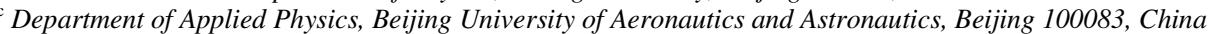

\begin{abstract}
Two different spatial levels are involved concerning damage accumulation to eventual failure. This can entail sample size $\ell(\sim \mathrm{cm})$ to characteristic microdamage size $c^{*}(\sim \mu \mathrm{m})$. Associated are three physical processes with three different rates, namely macroscopic elastic wave velocity a, nucleation and growth rates of microdamage $\mathrm{n}_{\mathrm{N}}{ }^{*}$ and $\mathrm{V}^{*}$.

It is found that the trans-scale length ratio $\mathrm{c}^{*} / \mathrm{L}$ does not directly affect the process. Instead, two independent dimensionless numbers: the trans-scale one $\mathrm{De}^{*}=\mathrm{ac}^{*} /\left(\ell \mathrm{V}^{*}\right)$ including the length ratio and the intrinsic one $\mathrm{D}^{*}=\mathrm{n}_{\mathrm{N}}{ }^{*} \mathrm{c}^{* 5} / \mathrm{V}^{*}$ including mesoscopic parameters only, play the key role in the process of damage accumulation to failure.

The above implies that there are three time scales involved in the process: the macroscopic imposed time scale $\mathrm{t}_{\mathrm{im}}=\ell / \mathrm{a}$ and two meso-scopic time scales, nucleation and growth of damage, $t_{N}=1 /\left(n_{N}^{*} c^{* 4}\right)$ and $t_{V}=c^{*} / V^{*}$. Clearly, the dimensionless number $D e^{*}=t_{V} / t_{i m}$ refers to the ratio of microdamage growth time scale over the macroscopically imposed time scale. So, analogous to the definition of Deborah number as the ratio of relaxation time over external one in rheology. Let De be the imposed Deborah number while De* represents the competition and coupling between the microdamage growth and the macroscopically imposed wave loading. In stress-wave induced tensile failure (spallation) $\mathrm{De}^{*}<1$, this means that microdamage has enough time to grow during the macroscopic wave loading. Thus, the microdamage growth appears to be the predominate mechanism governing the failure.

Moreover, the dimensionless number $D^{*}=t_{V} / t_{N}$ characterizes the ratio of two intrinsic mesoscopic time scales: growth over nucleation. Similarly let $\mathrm{D}^{*}$ be the "intrinsic Deborah number". Both time scales are relevant to intrinsic relaxation rather than imposed one. Furthermore, the intrinsic Deborah number $\mathrm{D}^{*}$ implies a certain characteristic damage. In particular, it is derived that $\mathrm{D}^{*}$ is a proper indicator of macroscopic critical damage to damage localization, like $\mathrm{D}^{*} \sim\left(10^{-3} \sim 10^{-2}\right)$ in spallation. More importantly, we found that this small intrinsic Deborah number $\mathrm{D}^{*}$ indicates the energy partition of microdamage dissipation over bulk plastic work. This explains why spallation can not be formulated by macroscopic energy criterion and must be treated by multi-scale analysis.
\end{abstract}

Keywords: Deborah numbers; Trans-scale coupling; Damage evolution; Failure.

\footnotetext{
* Corresponding author.

E-mail address: why@lnm.imech.ac.cn (H.Y. Wang).
} 


\section{Introduction}

From damage accumulation to failure, there prevails an important time-dependent phenomenon involving spallation in which failure occurs under transient loading like nano- to micro-seconds. Experimental observations suggest a time-integral criterion for spallation [1],

$\left(\sigma / \sigma^{*}-1\right)^{v} \times \Delta \mathrm{t}=\mathrm{K}$

where $\sigma$ and $\sigma^{*}$ are stress and a stress threshold respectively, $\Delta \mathrm{t}$ is the load duration, $v v$ and $\mathrm{K}$ are two parameters. This criterion indicates that the critical stress to spallation is no longer a material constant, but a variable depending on its loading duration. Furthermore, since the power exponent $v$ in the criterion is usually neither 1 nor 2, the criterion implies neither momentum nor energy criteria macroscopically [1-3]. Comprehensive and critical reviews on spallation have been made [4-6]. It is stressed that "the continuum models based on the statistical nucleation and growth of brittle and ductile fracture appear to be an attractive approach, especially with a framework which provides some forms of a continuum cumulativedamage description of the evolving fracture state" [6]. Recently, the work in [7] suggests that "dynamic failure by the growth and coalescence of grain-boundary microcracks involve the cooperative interactions of propagating cracks. Insight into such processes is required from the perspective of stochastic mechanics and from computer simulations of the debonding of assemblages of grains".

It follows that spallation is a typical process with coupled multiple space and time scales. At least, there are two length scales: the sample size at macroscopic level and the microdamage size at mesoscopic level. On the other hand, there are three time scales: the stress wave loading duration macroscopically, the mesoscopic nucleation time and growth time of microdamage. So, spallation represents an illustrative example with multiple space and time scales.

\section{Statistical Microdamage Mechanics}

The general evolution equation of microdamage number density is [8]

$\frac{\partial \mathrm{n}}{\partial \mathrm{t}}+\sum_{\mathrm{i}=1}^{\mathrm{I}} \frac{\partial\left(\mathrm{n} \cdot \mathrm{P}_{\mathrm{i}}\right)}{\partial \mathrm{p}_{\mathrm{i}}}=\mathrm{n}_{\mathrm{N}}$

where $t$ is time, $n_{N}$ is the nucleation rate of microdamage number density. $P_{i}=\dot{p}_{i}$, "." denotes the rate of variable $\mathrm{p}_{\mathrm{i}}$, which represents the state of microdamage. After taking the phase variable $\mathrm{p}$ as the current size of microdamage $\mathrm{c}$, i.e. $\mathrm{p}=\mathrm{c}$, we have obtained a general solution to microdamage number density $\mathrm{n}(\mathrm{t}, \mathrm{c} ; \sigma),[9-10]$ 


$$
\mathrm{n}(\mathrm{t}, \mathrm{c} ; \sigma)=\left\{\begin{array}{l}
\int_{0}^{\mathrm{c}} \frac{\mathrm{n}_{\mathrm{N}}\left(\mathrm{c}_{0} ; \sigma\right)}{\mathrm{V}\left(\mathrm{c}, \mathrm{c}_{0} ; \sigma\right)} \mathrm{dc}_{0}, \mathrm{c}<\mathrm{c}_{\mathrm{f}, 0} \\
\int_{\mathrm{c}_{0 \mathrm{f}}} \frac{\mathrm{n}_{\mathrm{N}}\left(\mathrm{c}_{0} ; \sigma\right)}{\mathrm{V}\left(\mathrm{c}, \mathrm{c}_{0} ; \sigma\right)} \mathrm{dc}_{0}, \mathrm{c}>\mathrm{c}_{\mathrm{f}, 0}
\end{array}\right.
$$

where $c_{o}$ is the nucleation size of microdamage and the time-dependent feature is expressed by the moving front of microdamage $c_{\text {of }}$ or $c_{f}$ in terms of the integral

$$
\mathrm{t}=\int_{c_{0 f}}^{\mathrm{c}} \frac{\mathrm{dc}}{\mathrm{V}\left(\mathrm{c}, \mathrm{c}_{\mathrm{o}} ; \sigma\right)}=\int_{\mathrm{c}_{0}}^{\mathrm{c}_{\mathrm{f}}} \frac{\mathrm{dc}}{\mathrm{V}\left(\mathrm{c}, \mathrm{c}_{\mathrm{o}} ; \sigma\right)}
$$

More importantly, there are two mesoscopic rate processes of microdamage involved in solution $(3): \mathrm{n}_{\mathrm{N}}\left(\mathrm{c}_{0} ; \sigma\right)$ is the nucleation rate of microdamage number density and $\mathrm{V}=\mathrm{V}\left(\mathrm{c}, \mathrm{c}_{0} ; \sigma\right)$ is the growth rate of microdamage.

The relation between continuum damage $\mathrm{D}$ and the number density of microdamage $n$ is

$\mathrm{D}(\mathrm{t}, \mathbf{x})=\int_{0}^{\infty} \mathrm{n}(\mathrm{t}, \mathbf{x}, \mathrm{c}) \cdot \tau \cdot \mathrm{dc}$

where $\tau \sim \mathrm{c}^{3}$ is the failure volume of an individual microdamage with size $\mathrm{c}$, [11-12]. Then, the statistical evolution equation of microdamage number density in Eq. (2) can be converted to the continuum damage field equation by integration under proper boundary conditions and its the one-dimensional Lagrangian form is [11-12].

$$
\begin{aligned}
& \frac{\partial \mathrm{D}}{\partial \mathrm{T}}+\mathrm{D} \frac{\rho}{\rho_{0}} \frac{\partial \mathrm{v}}{\partial \mathrm{X}}=\mathrm{f} \\
& =\int_{0}^{\infty} \mathrm{n}_{\mathrm{N}}(\mathrm{c} ; \sigma) \tau(\mathrm{c}) \mathrm{dc} \cdot\left\{1+\frac{\int_{0}^{\infty} \mathrm{n}_{\mathrm{N}}\left(\mathrm{c}_{0} ; \sigma\right) \int_{\mathrm{c}_{0}}^{\mathrm{c}_{\mathrm{f}}} \tau^{\prime}(\mathrm{c}) \mathrm{dcdc}_{0}}{\int_{0}^{\infty} \mathrm{n}_{\mathrm{N}}(\mathrm{c} ; \sigma) \tau(\mathrm{c}) \mathrm{dc}}\right\}
\end{aligned}
$$

where $\mathrm{f}$ is the dynamic function of damage, which represents the statistical average effects of nucleation and growth of microdamage on continuum damage evolution and $\tau^{\prime}=\mathrm{d} \tau / \mathrm{dc}$. Combining the damage field relation in Eq. (6) with the following conventional field equations of continuum, momentum and energy,

$$
\begin{aligned}
& \frac{\partial \varepsilon}{\partial T}=\frac{\partial v}{\partial X} \\
& \rho_{0} \frac{\partial v}{\partial T}=\frac{\partial \sigma}{\partial X}
\end{aligned}
$$


$\rho_{0} \mathrm{c}_{\mathrm{v}} \frac{\partial \theta}{\partial \mathrm{T}}=\frac{\partial \mathrm{e}_{\text {diss }}}{\partial \mathrm{T}}+\lambda \frac{\partial^{2} \theta}{\partial \mathrm{X}^{2}}$

a coupled trans-scale framework is formed for the damage evolution [13-14], where $\rho_{0}$ is density of intact material, $\lambda$ is heat conductivity, $\theta$ is temperature and $\mathrm{e}_{\mathrm{diss}}$ is the energy dissipated in the material element.

\section{Deborah Numbers, Coupling Multiple Space and Time Scales}

In accord with $\Pi$ theorem in dimensional analysis, 10 parameters involved in spallation (see Table 1), can form 6 independent dimensionless numbers. However, when Eqs. (6-9) are non-dimensionalized it is found that there are 5 independent quantities only.

Table 1. 8 parameters involved in spallation

\begin{tabular}{|c|c|c|c|}
\hline & parameter & symbol & dimension \\
\hline \multirow{4}{*}{ macroscopic } & Sample size & $\ell$ & $\mathrm{L}$ \\
\cline { 2 - 4 } & Density & $\rho$ & $\mathrm{ML}^{-3}$ \\
\cline { 2 - 4 } & Elastic wave speed & $\mathrm{a}$ & $\mathrm{LT}^{-1}$ \\
\cline { 2 - 4 } & Characteristic stress & $\sigma_{\mathrm{Y}}$ & $\mathrm{ML}^{-1} \mathrm{~T}^{-2}$ \\
\cline { 2 - 4 } & Impact velocity & $\mathrm{v}_{\mathrm{f}}$ & $\mathrm{LT}^{-1}$ \\
\cline { 2 - 4 } & Heat conductivity & $\mathrm{MLT}^{-3} \theta^{-1}$ \\
\cline { 2 - 4 } & Heat capacity & $\mathrm{c}_{\mathrm{V}}$ & $\mathrm{L}^{2} \mathrm{~T}^{-2} \theta^{-1}$ \\
\hline \multirow{3}{*}{ mesoscopic } & Characteristic nucleation rate of microdamage & $\mathrm{n}_{\mathrm{N}}{ }^{*}$ & $\mathrm{~L}^{-4} \mathrm{~T}^{-1}$ \\
\cline { 2 - 4 } & Characteristic growth rate of microdamage. & $\mathrm{V}^{*}$ & $\mathrm{LT}^{-1}$ \\
\cline { 2 - 4 } & Characteristic size of microdamage & $\mathrm{c}^{*}$ & $\mathrm{~L}$ \\
\hline
\end{tabular}

In the 5 dimensionless numbers, there are 3 conventional macroscopic ones: the well-known Mach number, damage number and Fourier number, related to continuum, momentum and energy equations respectively. Consider the other two trans-scale dimensionless numbers: the imposed Deborah number $\mathrm{De}^{*}=\frac{\mathrm{ac}^{*}}{\ell \mathrm{V}^{*}}$ and the intrinsic Deborah number $\mathrm{D}^{*}=\frac{\mathrm{n}_{\mathrm{N}}{ }^{*} \mathrm{c}^{{ }^{* 5}}}{\mathrm{~V}^{*}}$.

For a group of $\mathrm{Al}$ alloy spallation tests, $\ell \sim(5-10) \mathrm{mm}, \mathrm{v}_{\mathrm{f}} \sim 10^{2} \mathrm{~ms}^{-1}, \mathrm{c}^{*} \sim$ $4.27 \cdot 10^{-6} \mathrm{~m}, \mathrm{~V}^{*} \sim 5.96 \mathrm{~ms}^{-1}$ and $\mathrm{n}_{\mathrm{N}}{ }^{*} \sim 5.22 \cdot 10^{4} \mathrm{~mm}^{-3} \mu \mathrm{m}^{-1} \mu \mathrm{s}^{-1}$, derivation leads to $\mathrm{De}^{*} \sim 1$ and $\mathrm{D}^{*} \sim\left(10^{-2} \sim 10^{-3}\right)^{[10-12]}$.

Noticeably, the ratio of two length scales on meso- and macro-levels $\mathrm{c}^{*} / \ell$ does not appear here. Actually, the imposed Deborah number $\mathrm{De}^{*}=\frac{\mathrm{ac}^{*}}{\ell \mathrm{V}^{*}}$ is a combination of two ratios: the size scale ratio $\mathrm{c}^{*} / \ell$ and the ratio of two velocities $\mathrm{V}^{*} / \mathrm{a}$. Also, the imposed Deborah number $\mathrm{De}^{*}$ is a unique trans-scale dimensionless parameter, since elastic wave speed a and sample size $\ell$ are macroscopic parameters whereas microdamage size $\mathrm{c}^{*}$ and microdamage growth rate $\mathrm{V}^{*}$ are mesoscopic ones. This is 
very different from all other dimensionless parameters. On the other hand, $\mathrm{De}^{*}=\mathrm{t}_{\mathrm{V}} / \mathrm{t}_{\text {im }}$ refers to the ratio of microdamage growth time scale $\mathrm{t}_{\mathrm{V}}=\mathrm{c}^{*} / \mathrm{V}^{*}$ over the macroscopically imposed time scale $t_{\mathrm{im}}=\ell / \mathrm{a}$. This is why we call it Deborah number.

The intrinsic Deborah number $\mathrm{D}^{*}=\frac{\mathrm{n}_{\mathrm{N}}{ }^{*} \mathrm{c}^{* 5}}{\mathrm{~V}^{*}}$ characterizes the rate ratio of the two intrinsic mesoscopic processes: nucleation over growth. Actually, $D^{*}=t_{v} / t_{N}$, where $t_{V}=c^{*} / V^{*}$ and $t_{N}=\left(n_{N}^{*} c^{* 4}\right)^{-1}$ are the growth and nucleation time scales respectively. Note that the characteristic nucleation time $t_{N}$ is not the time needed for an individual microdamage to nucleate, but the time to form a characteristic nucleated damage fraction, since $\dot{\mathrm{D}}_{\mathrm{N}}^{*}$ means the damage fraction merely due to nucleation in unit time.

$$
\begin{aligned}
\mathrm{t}_{\mathrm{N}}^{*} & =1 / \mathrm{n}_{\mathrm{N}}^{*} \mathrm{c}^{* 4}=1 /\left[\left(\mathrm{n}_{\mathrm{N}}^{*} \mathrm{c}^{*}\right) \mathrm{c}^{* 3}\right] \\
& =1 /\left(\mathrm{N}_{\mathrm{N}}{ }^{*} \mathrm{c}^{* 3}\right)=1 / \dot{\mathrm{D}}_{\mathrm{N}}^{*}
\end{aligned}
$$

Above all, in the case study of spallation, there are three time scales: the macroscopic imposed time scale $\mathrm{t}_{\mathrm{im}}=\ell / \mathrm{a} \sim 10^{-6} \mathrm{~s}$ and two meso-scopic time scales, growth time scale $\mathrm{t}_{\mathrm{V}}=\mathrm{c}^{*} / \mathrm{V}^{*} \sim 10^{-6} \mathrm{~s}$ and the nucleation time scale $\mathrm{t}_{\mathrm{N}}=\left(\mathrm{n}_{\mathrm{N}}^{*} \mathrm{C}^{* 4}\right)^{-1} \sim 10^{-3} \mathrm{~s}$. These lead to $\mathrm{De}^{*} \sim 1$ and $\mathrm{D}^{*} \sim\left(10^{-2} \sim 10^{-3}\right)$ too. However, this is not the whole story of Deborah numbers.

\section{The Significance of Deborah Numbers in Failure}

\subsection{Imposed Deborah number governs the failure process}

Note that the imposed Deborah number $D e^{*}=t_{V} / t_{i m}$ represents the competition and coupling between the microdamage growth and the macroscopically imposed wave loading. Also, in the concerned spallation case $\mathrm{De}^{*} \sim 1$, this indicates that microdamage has enough time to grow during the macroscopic wave loading and then microdamage growth may be the predominate mechanism governing spallation.

\subsection{Intrinsic Deborah number signifies characteristic damage and damage localization}

Turn to the intrinsic Deborah number $\mathrm{D}^{*}$. Firstly, D*implies a certain characteristic damage, since Eq. (5) can be written as 
$\mathrm{D}=\int_{0}^{\infty} \mathrm{n}(\mathrm{c}) \cdot \tau \cdot \mathrm{dc}=\frac{\mathrm{n}_{\mathrm{N}}^{*} \mathrm{c}^{* 5}}{\mathrm{~V}^{*}} \int_{0}^{\infty} \overline{\mathrm{n}}_{\mathrm{N}} \mathrm{d} \overline{\mathrm{c}}_{0} \int_{\overline{\mathrm{c}}_{0}}^{\overline{\mathrm{c}}_{\mathrm{f}}} \frac{\overline{\mathrm{c}}^{3}}{\overline{\mathrm{V}}} \mathrm{d} \overline{\mathrm{c}}$

All variables with bar above denote non-dimensionalized and normalized ones, hence the preceding dimensionless combination, i.e. the intrinsic Deborah number $\mathrm{D}^{*}$, represents the magnitude of damage $\mathrm{D}$. In the concerned spallation, this means a characteristic damage is of $\mathrm{D}^{*} \sim\left(10^{-2} \sim 10^{-3}\right)$.

Also, $D^{*}$ is a proper indicator of critical damage to localization. Damage localization can be formulated as follows ${ }^{[15]}$

$$
\frac{\left[\frac{\partial\left(\frac{\partial \mathrm{D}}{\partial \mathrm{X}}\right)}{\partial \mathrm{T}}\right]}{\left(\frac{\partial \mathrm{D}}{\partial \mathrm{X}}\right)} \geq \frac{\left(\frac{\partial \mathrm{D}}{\partial \mathrm{T}}\right)}{\mathrm{D}}
$$

It has been derived that provided the dynamic function of damage can be expressed by

$$
\mathrm{f}=\mathrm{f}(\mathrm{D}, \sigma)
$$

Under a certain approximation the damage localization condition (12) can be expressed by the following inequality [15],

$\mathrm{f}_{\mathrm{D}} \geq \frac{\mathrm{f}}{\mathrm{D}}$

Where $f_{D}=\frac{\partial f}{\partial D}$. Obviously, this condition (14) represents an intrinsic feature and irrelevant to any specific conditions of tests.

Combining the damage localization condition in Eq. (14), the definition of dynamic function of damage in Eq. (6) and the expression of damage in Eq. (5), a critical damage to localization can be derived:

$$
\begin{aligned}
\mathrm{D}_{\mathrm{c}} & \approx \frac{\mathrm{n}_{\mathrm{N}}{ }^{*} \mathrm{c}^{* 5}}{\mathrm{~V}^{*}} \cdot \frac{\int_{0}^{\infty} \bar{\tau}\left(\overline{\mathrm{c}}_{\mathrm{f}}\right) \overline{\mathrm{n}}_{\mathrm{N}}\left(\overline{\mathrm{c}}_{0}\right) \mathrm{d} \overline{\mathrm{c}}_{0} \cdot \int_{0}^{\infty} \frac{\bar{\tau}\left(\overline{\mathrm{c}}_{\mathrm{f}}\right) \overline{\mathrm{n}}_{\mathrm{N}}\left(\overline{\mathrm{c}}_{0}\right)}{\overline{\mathrm{V}}\left(\overline{\mathrm{c}}_{0}, \mathrm{c}_{\mathrm{f}}\right)} \mathrm{d} \overline{\mathrm{c}}_{0}}{\int_{0}^{\infty} \bar{\tau}^{\prime}\left(\overline{\mathrm{c}}_{\mathrm{f}}\right) \overline{\mathrm{n}}_{\mathrm{N}}\left(\overline{\mathrm{c}}_{0}\right) \mathrm{d} \overline{\mathrm{c}}_{0}} \\
& =\mathrm{D}^{*} \cdot \mathrm{O}(1)
\end{aligned}
$$

This once more is the intrinsic Deborah number D*.

As above, the dimensionless combination preceding the normalized integrals indicates the magnitude of the critical damage to localization. Compare the obtained 
result with experiments. As noted before, experiments gave $\mathrm{D}^{*}=\frac{\mathrm{n}_{\mathrm{N}}{ }^{*} \mathrm{c}^{* 5}}{\mathrm{~V}^{*}} \sim\left(10^{-2} \sim\right.$ $10^{-3}$ ). According to the localization condition in Eq. (14), there results in the critical damage to localization $D_{c} \sim 4^{*} 10^{-3}[12]$. Clearly, the intrinsic Deborah number $\mathrm{D}^{*}$ does characterizes the magnitude of the critical damage $\mathrm{D}_{\mathrm{c}}$. Some simulations (Fig. 1) demonstrate that the intrinsic Deborah number does signify a certain characteristics in damage localization.

In the numerical study, a fixed $\mathrm{De}=\mathrm{De}^{*} / \mathrm{D}^{*}=65.9$ is taken. The most localized distribution occurs in the case of $\left(\mathrm{De}^{*}=0.151\right.$ equivalent to $\left.\mathrm{D}^{*}=0.0023\right)$, whilst the case $\left(\mathrm{De}^{*}=0.415\right.$ equivalent to $\left.\mathrm{D}^{*}=0.0063\right)$ demonstrates hardly localized distribution. This is in agreement with the analysis that lower $\mathrm{D}^{*}$ indicates lower threshold of critical damage to localization. In Fig. $1, \ell$ is the plate thickness (sample size).

\subsection{Intrinsic Deborah number signifies partition of energy dissipation}

The energy partition will be clarified by examining the energy equation [16-17]. After splitting the dissipation term into damage and plastic ones, Eq. (9) may be rewritten as

$\rho_{0} \mathrm{c}_{\mathrm{v}} \frac{\partial \theta}{\partial \mathrm{T}}=\sigma \frac{\partial \varepsilon^{\mathrm{p}}}{\partial \mathrm{T}}+\gamma \frac{\partial \Sigma}{\partial \mathrm{T}}+\lambda \frac{\partial^{2} \theta}{\partial \mathrm{X}^{2}}$

where $\Sigma$ and $\gamma$ are the total surface area of microdamage and corresponding equivalent surface energy in unit volume respectively. Certainly, the partition of plastic dissipation (the first term on the right hand side) and the damage one (the second term) are of our interest. Again, the dimensionless energy equation is:

$\frac{\partial \bar{\theta}}{\partial \overline{\mathrm{T}}}=\bar{\sigma} \frac{\partial \bar{\varepsilon}^{\mathrm{p}}}{\partial \overline{\mathrm{T}}}+\mathrm{D}^{*} \frac{\partial \bar{\Sigma}}{\partial \overline{\mathrm{T}}}+\Psi \frac{\partial^{2} \bar{\theta}}{\partial \overline{\mathrm{Y}}^{2}}$

As before, all variables with bars are dimensionless and normalized, i.e. in $\mathrm{O}(1)$. The last term indicates the heat transfer and $\Psi$ is a dimensionless number relevant to Fourier number. For aluminum, $\lambda=238 \mathrm{~W} / \mathrm{m} \cdot \mathrm{K}, \rho_{0} \sim 2700 \mathrm{Kg} / \mathrm{m}^{3}, \mathrm{c}_{\mathrm{V}} \sim 903 \mathrm{~J} / \mathrm{kg} \cdot \mathrm{K}$, $\mathrm{a} \sim 5000 \mathrm{~m} / \mathrm{s}, \Psi=\frac{\lambda}{\rho_{0} \mathrm{c}_{\mathrm{v}} \ell \mathrm{a}}=\frac{\lambda / \rho_{0} \mathrm{v}_{\mathrm{v}}}{\ell \mathrm{a}}=\frac{\kappa / \mathrm{a}}{\ell}=10^{-3}<<1$. This implies that spallation appears to be adiabatic.

Now, the energy dissipation involved in spallation consists of two parts only: plastic dissipation and damage dissipation. Examine the term of damage dissipation in more details. Approximately, $\gamma$ can be estimated with $\left(\sigma_{\mathrm{Y}} \varepsilon_{\mathrm{Y}} \mathrm{c}^{*}\right)$ and $\Sigma$ with $\left(\mathrm{n}_{\mathrm{N}}{ }^{*} \mathrm{c}^{*}{ }^{*} / \mathrm{V}^{*}\right)$, whereas the plastic dissipation with $\sigma_{\mathrm{Y}} \varepsilon_{\mathrm{Y}}$. therefore, the dimensionless and normalized combination preceding the damage dissipation term 
$\gamma \frac{\partial \Sigma}{\partial \mathrm{T}}$ is $\left(\sigma_{\mathrm{Y}} \varepsilon_{\mathrm{Y}} \mathrm{c}^{*}\right)\left(\mathrm{n}_{\mathrm{N}}{ }^{*} \mathrm{c}^{*} / \mathrm{V}^{*}\right) /\left(\sigma_{\mathrm{Y}} \varepsilon_{\mathrm{Y}}\right)=\mathrm{n}_{\mathrm{N}}{ }^{*} \mathrm{c}^{* 5} / \mathrm{V}^{*}=\mathrm{D}^{*}$ as shown in Eq. (17).

It becomes clear that the energy dissipation involved in spallation consists of two very different parts: plastic dissipation in $\mathrm{O}(1)$ whilst damage dissipation in $\mathrm{O}\left(\mathrm{D}^{*}\right)$, see Eq. (17). It can be said that the temperature rise in spallation is mainly due to plastic dissipation, since $\mathrm{D}^{*}<<1$. Fig. 2 gives a numerical result of the energy partition in spallation. One can notice that the damage dissipation varies around the magnitude of the intrinsic Deborah number $\mathrm{D}^{*}$.

It is clear from Fig. 2(a) that the temperature increments induced by damage is much less that that induced by plastic dissipation. And the ratio of temperature increments due to damage over plastic dissipation varies around the value of $\mathrm{D}^{*}$, as shown in Fig. 2(b).

\section{Conclusion}

Two independent dimensionless numbers: the trans-scale imposed Deborah number $\mathrm{De}^{*}=\frac{\mathrm{ac}^{*}}{\ell \mathrm{V}^{*}}$ and the intrinsic Deborah number $\mathrm{D}^{*}=\frac{\mathrm{n}_{\mathrm{N}}{ }^{*} \mathrm{c}^{{ }^{5}}}{\mathrm{~V}^{*}}$ govern the process of damage accumulation to failure.

The trans-scale imposed Deborah number $\mathrm{De}^{*}=\frac{\mathrm{ac}^{*}}{\ell \mathrm{V}^{*}}$ represents the competition and coupling between the microdamage growth and the macroscopically imposed wave loading. In spallation $\mathrm{De}^{*}<1$ means that micro-damage has enough time to grow during the macroscopic wave loading. Thus, the microdamage growth appears to be the predominate mechanism governing the failure.

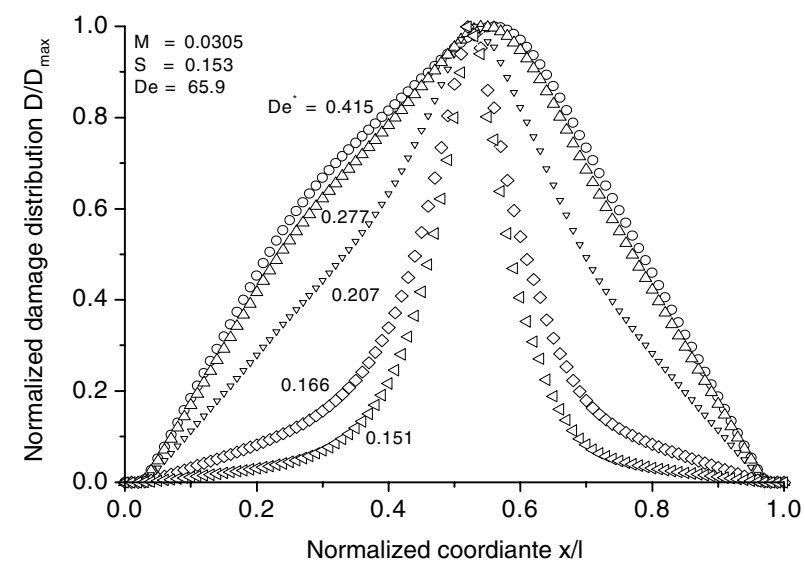

Fig. 1. The spatial distribution of damage normalized by its maximum. 


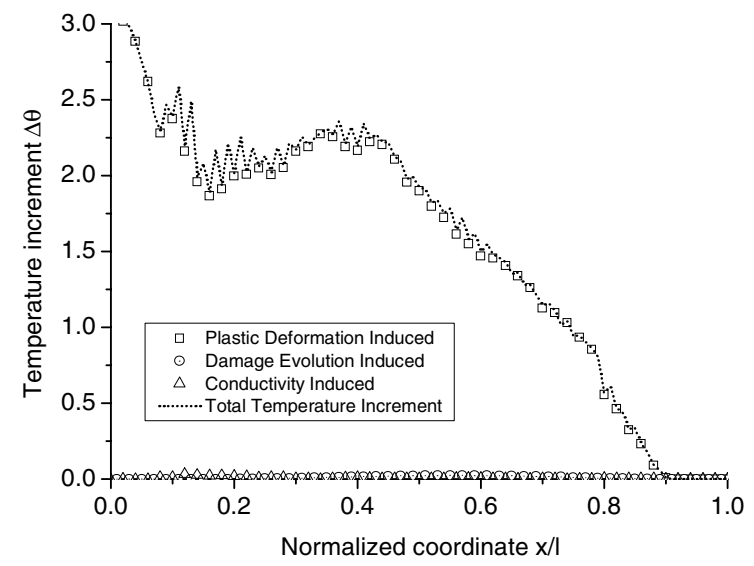

(a) Temperature increment

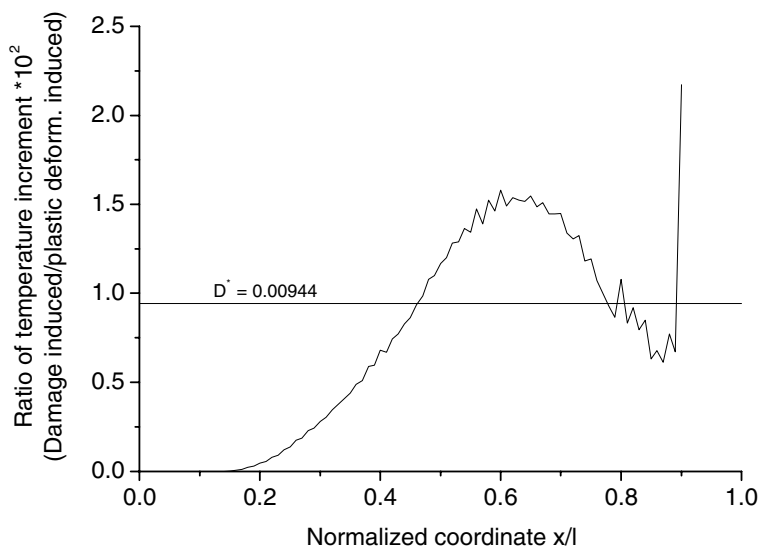

(b) Ratio of temp. increment

Fig. 2. The spatial distribution of temperature rise.

The intrinsic Deborah number $\mathrm{D}^{*}=\frac{\mathrm{n}_{\mathrm{N}}{ }^{*} \mathrm{c}^{* 5}}{\mathrm{~V}^{*}}$ characterizes the ratio of microdamage growth rate over nucleation. The intrinsic Deborah number $\mathrm{D}^{*}$ implies a certain characteristic damage and is a proper indicator of critical damage to damage localization. More importantly, this small intrinsic Deborah number $D^{*} \sim\left(10^{-2}\right.$ $\sim 10^{-3}$ ) indicates the energy partition of microdamage dissipation over bulk plastic work in spallation. 


\section{Acknowledgements}

This work is granted by the National Natural Science Foundation of China (NSFC No. 10432050, 10432040, 10372012, 10302029, 10472118) and Chinese Academy of Sciences.

\section{References}

[[1] Tuler FR and Butcher BM. A criterion for the time dependence of dynamic fracture. Int $\mathbf{J}$ Fracture Mech 1968; 4(4): 431-437.

[2] Shen LT, Zhao SD, Bai YL and Luo LM. Experimental Study on the Criteria and Mechanism of Spallation in an Al Alloy. Int J Impact Eng 1992; 12(1): 9-19.

[3] Zhu ZX. private communication 1985.

[4] Curran DR, Seaman L, Shockey DA. Dynamic failure of solids. Phys Rep 1987; 147: 253-388.

[5] Meyers MA. Dynamic Behaviour of Materials. New York: John Wiley \& Sons Inc., 1994.

[6] Grady DE. The spall strength of condensed matter. J Mech Phys Solids 1988; 36(3): 353-384.

[7] Clifton RJ. Response of materials under dynamic loading. Int J Solids Struct 2000; 37(1-2): 105-113.

[8] Bai YL, Ke FJ, Xia MF. Formulation of statistical evolution of microcracks in solids. Acta Mechanica Sinica 1991; 7: 59-66.

[9] Ke FJ, Bai YL, and Xia MF. Evolution of ideal micro-crack system. Science in China Ser. A 1990; 33: 1447-1459.

[10] Han WS, Xia MF, Shen LT and Bai YL. Statistical Formulation and Experimental Determination of Growth Rate of Micrometer Cracks under Impact Loading. Int J Solids Struct 1997; 34: 2905-2925.

[11] Xia MF, Han WS, Ke FJ and Bai YL. Statistical meso-scopic damage mechanics and damage evolution induced catastrophe. Advances in Mechanics (in Chinese) 1995; 25: 1-40, 145-173.

[12] Bai YL, Bai J, Li HL, Ke FJ, Xia MF. Damage evolution, localization and failure of solids subjected to Impact Loading. Int J Impact Engng 2000; 24: 685-701.

[13] Bai YL, Xia, MF, Wei YJ, Ke FJ. Non-Equilibrium Evolution of Collective Microdamage and Its Coupling with Mesoscopic Heterogeneities and Stress Fluctuations. In: Horie Y, Davison L, Thadhani NN, editors. High Pressure Shock Compression of Solids VI - Old Paradigms and New Challenges. Springer-Verlag, 2002, p. 255-278.

[14] Bai YL, Xia MF, Ke FJ and Li HL (2002), Closed trans-scale statistical microdamage mechanics, Acta Mech. Sinica, 18(1)1-17.

[15] Bai YL, Xia MF, Ke FJ, Li HL (1997). Damage field equation and criterion for damage localization. In: Dordrecht, Wang R, editors. Rheology of Bodies with Defects. Kluwer Academic Publishers, 1997, p. 55-66.

[16] Wang HY, Bai YL, Wei YJ. Analysis of spallation based on trans-scale formulation of damage evolution. 2nd int Conf. Structural Stability and Dynamics, 2002.

[17] Wang HY, Bai YL and Wei YJ. Analysis of spallation based on trans-scale formulation of damage evolution. Acta Mech Sinica 2004; 20(4): 400-407. 